\title{
Locally Produced IGF-1 Promotes Hypertrophy of the Ligamentum Flavum via the mTORC1 Signaling Pathway
}

\author{
Bin Yann ${ }^{a, b, c}$ Minjun Huang ${ }^{a, b, c} \quad$ Canjun Zeng, ${ }^{a, b, c} \quad N^{2}$ Yao $^{d}$ Jie Zhang ${ }^{a, b, c}$ Bo Yan ${ }^{a, b, c}$ \\ Huaji Jianga, ${ }^{a, b, c}$ Xinggui Tian ${ }^{a, b, c}$ Xiang Ao $o^{a, b, c}$ Huiyu Zhao ${ }^{a, b, c}$ Wen Zhou ${ }^{a, b, c}$ \\ Jun Chu ${ }^{a, b, c}$ Liping Wang ${ }^{e}$ Cory J. Xian ${ }^{e}$ Zhongmin Zhang ${ }^{a, b, c}$ Liang Wang \\ aDepartment of Orthopedics, the Third Affiliated Hospital of Southern Medical University, Guangzhou, \\ bOrthopaedic Hospital of Guangdong Province, Guangzhou, 'Academy of Orthopaedics of Guangdong \\ Province, Guangzhou, dGuangdong Food and Drug Vocational-technical School; Guangzhou, China, \\ eSansom Institute for Health Research, School of Pharmacy and Medical Sciences, University of South \\ Australia, Adelaide, Australia
}

\section{Key Words}

IGF-1 • Hypertrophy • Ligamentum flavum • mTORC1 • Signaling

\begin{abstract}
Background/Aims: Narrowing of the lumbar spinal canal is a condition called lumbar spinal stenosis (LSS) and is a high-morbidity problem in the elderly. LSS is commonly caused by hypertrophy of the ligamentum flavum (HLF). Previous studies showed that fibrosis of the ligamentum flavum (LF) largely contributed to HLF. However, the underlying pathomechanism remains unclear. Insulin-like growth factor-1 (IGF-1) is known to have an intimate relationship with fibrosis in various tissues. Nevertheless, currently, there are few studies regarding IGF1 in HLF. In this study, we investigated the role of IGF-1 in HLF and its potential molecular mechanism of action. Methods: First, the IGF-1, phosphorylation of IGF-1 receptor (pIGF$1 R)$, phosphorylation of AKT (pAKT), phosphorylation of S6(pS6), collagen I and collagen III expression levels were examined via immunohistochemistry and Western blotting in LF tissues from patients with LSS or Non-LSS. Second, primary LF cells were isolated from adults with a normal LF thickness and were cultured with different concentrations of IGF-1 with or without NVP-AEW541/rapamycin. Results: The results showed that IGF-1, pIGF-1R, pAKT, pS6, collagen I and collagen III protein expression in the LSS group was significantly higher than that in the Non-LSS group. Meanwhile, pIGF-1R, pAKT, pS6, collagen I and collagen III protein expression was significantly enhanced in LF cells after IGF-1 exposure, which can be notably blocked by NVP-AEW541. In addition, pS6, collagen I and collagen III protein expression was blocked by rapamycin. Conclusions: Enhanced IGF-1 promotes the synthesis of collagen I and collagen III via the mTORC1 signaling pathway, which eventually contributes to hypertrophy of the ligamentum flavum.

B. Yan, M. Huang and C. Zeng contributed equally to this work.

\begin{tabular}{ll}
\hline Liang Wang & Department of Orthopedics, The Third Affiliated Hospital of Southern Medical University \\
and Zhongmin Zhang & Guangzhou, 510630, Guangdong (China) \\
& Tel. (86)2062784300, Fax (86)2062784308, E-Mail liang091@aliyun.com, nfzzm@163.com
\end{tabular}
\end{abstract}




\section{Introduction}

Lumbar spinal stenosis (LSS) has a high rate of morbidity in elderly patients [1-3]. LSS can lead to severe low back pain, leg pain, intermittent claudication and disability [4, 5]. There are several pathogenic factors that lead to LSS, such as disc degeneration, facet degeneration, and hypertrophy of the ligamentum flavum (HLF)[6]. Among these pathogenic factors, HLF plays a vital role in LSS[7-10]. Previous studies have indicated that fibrosis of the ligamentum flavum stimulated by chronic inflammation is considered to be the most important pathological process of HLF $[11,12]$. In the hypertrophied ligamentum flavum, severe fibrosis was observed. Fibrosis was primarily caused by increased collagen I and collagen III[13-15]. Meanwhile, most investigators accept the theory that mechanical stress alteration of the ligamentum flavum stimulates the release of pro-inflammatory cytokines and growth factors, which ultimately induce fibrosis of the ligamentum flavum $[13,16,17]$.

Insulin-like growth factor-1 (IGF-1) is an important growth factor in the mammalian tissue microenvironment and plays an important role in tissue fibrosis by activating the mTORC1 signaling pathway [18-20]. In the skeletal system, the release of IGF-1 has been demonstrated to correlate with immediate mechanical stress [21, 22]. Under mechanical loading, IGF-1 is released into the matrix, which induces downstream production of collagen fibers and, ultimately, results in fibrosis and hypertrophy of different tissues [23-27]. However, despite these previous studies on the potential role of IGF-1 in tissue fibrosis and hypertrophy, the interconnection between IGF-1 and HLF has not been thoroughly explored.

In the current work, we hypothesized that IGF-1 may promote the production of collagen fibers that contribute to HLF by activating the mTORC1 signaling pathway. To verify our hypothesis, we first evaluated the protein expression of IGF-1, mTORC1-signaling-pathwayrelated proteins (pIGF-1R, pAKT and pS6), collagen I and collagen III in LF tissues. Next, to investigate the potential molecular mechanism, we conducted in vitro studies on isolated primary LF cells.

\section{Materials and Methods}

\section{Ligamentum flavum tissue samples}

Ligamentum flavum tissue samples were collected from twenty patients (10 males and 10 females, average age: 59.60 years) who underwent decompressive laminectomy for LSS. Meanwhile, twenty patients (8 males and 12 females, average age: 56.05 years old) without LSS (Non-LSS) who underwent a similar surgery were selected as the control group. The ligamentum flavum tissues were sampled from the L4/5 segments and rinsed in physiological saline 3 times. Part of each sample was fixed in 4\% paraformaldehyde for hematoxylin and eosin (H\&E) staining, Masson's trichrome staining and immunohistochemical analysis. The remaining samples were stored immediately in liquid nitrogen for subsequent Western blot analysis.

Informed consent was obtained from each patient, and the experiments performed in this study were approved by the Institutional Ethics Review Board of the Third Affiliated Hospital of Southern Medical University, Guangzhou, Guangdong, China.

\section{Measurement of LF thickness}

LF thickness was measured by obtaining preoperative magnetic resonance images (MRI, Philips, Amsterdam, Netherlands) from all 40 patients. On axial T2-weighted images through the facet joint [28, 29], the maximum thickness of the LF was measured by an experienced spine surgeon using the Picture Archiving and Communication Systems (PACS) software (The Third Affiliated Hospital of Southern Medical University, Guangzhou, China). Each ligament flavum was measured three times, and the average value was taken as the final LF thickness (Fig. 1).

Fibrosis evaluation of $L F$

LF tissue samples were fixed in $4 \%$ paraformaldehyde for $48 \mathrm{~h}$ before being processed and were embedded into paraffin blocks. The samples were cut into $4 \mu \mathrm{m}$ thick sections using a paraffin microtome 


\section{Cellular Physiology Cell Physiol Biochem 2018;48:293-303 \begin{tabular}{l|l} 
DOI: 10.1159/000491729 & O 2018 The Author(s). Published by S. Karger AG, Basel \\
www.karger.com/cpb
\end{tabular}}

(RM2125 RTS, Leica, Wetzlar, Germany). The sections were stained using H\&E staining and Masson's trichrome staining kits (JianChen, Nanjing, China). H\&E staining showed the morphology and structure of the LF. Simultaneously, the degree of LF fibrosis was evaluated according to the results of Masson's trichrome staining. Three randomly selected fields were observed under 200x magnification of each sample section to determine the grade of fibrosis. Similar to previous research [14], the degree of LF fibrosis was classified into 5 grades: Grade $0, \leq 20 \%$ of the area of the sample was stained blue; Grade 1, between $21 \%$ and $40 \%$ of the area of the sample was stained blue; Grade 2,

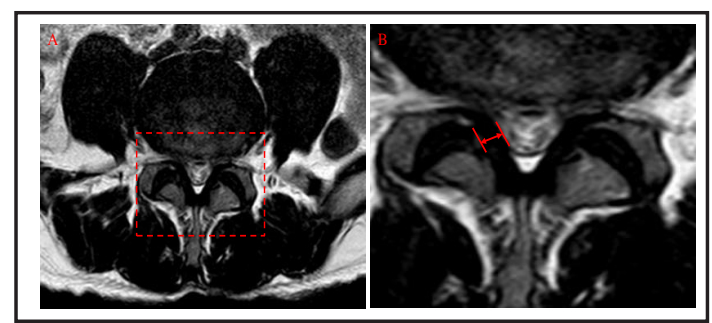

Fig. 1. Measurement of the ligamentum flavum thickness by MRI. (A) The target area was selected for analysis; (B) the arrow represents the ligamentum flavum thickness at the facet joint level. between $41 \%$ and $60 \%$ of the area of the sample was stained blue; Grade 3, between $61 \%$ and $80 \%$ of the area of the sample was stained blue; and Grade 4, over $81 \%$ of the whole area of the sample was stained blue.

\section{Immunohistochemical analyses}

$\mathrm{LF}$ tissue sections were deparaffinized and incubated for 10 minutes in $3 \% \mathrm{H}_{2} \mathrm{O}_{2}$ to quench endogenous peroxidase activity. For antigen retrieval, the sample sections in citrate buffer were heated for $16 \mathrm{~h}$ at $60^{\circ} \mathrm{C}$. Next, the sample sections were washed 3 times for 5 minutes each using phosphate-buffered saline (PBS). Non-specific binding was blocked for 30 minutes with 5\% horse serum. Primary antibodies against IGF1 (1:200, Abcam, Cambridge, UK), pIGF-1R (1:100, Abcam), pAKT (1:100, Abcam), pS6 (1:200, ABclonal, Boston, MA), collagen I (1:100, Abcam) and collagen III (1:100, Abcam) were applied overnight at $4^{\circ} \mathrm{C}$. After being washed 3 times for 5 minutes each with PBS, tissue sections were incubated with a goat-antirabbit horseradish peroxidase-conjugated secondary antibody (1:100, RayBiotech, Beijing, China) for 1.5 $\mathrm{h}$ at room temperature, and the immunostain signal was developed using 3, 3'-diaminobenzidine (DAB, ZSGB-Bio, Beijing, China). The numbers of positive cells (stained brown) were counted by 3 volunteers in a double-blinded manner in 5 randomly selected fields $(400 \times)$ using the ImagePro 4.5 software (Media Cybernetics, Rockville, MD).

\section{Transmission electron microscopy detection}

Ligamentum flavum tissues were cut into blocks of approximately $1.0 \mathrm{~mm}^{3}$ and were fixed in $2.5 \%$ glutaraldehyde for $4 \mathrm{~h}$. The samples were dehydrated with propylene oxide and were embedded in Epon. Finally, $100 \mathrm{~nm}$ thick ultrathin sections were cut using an ultramicrotome (UC7, Leica, Wetzlar, Germany), stained with $2 \%$ uranyl acetate, and observed under a transmission electron microscope (H-7500, Hitachi Technology, Tokyo).

\section{Culture and identification of LF cells}

LF samples of normal thickness were aseptically obtained from 8 adult patients who underwent spinal surgery. After washing in physiological saline 3 times, the LF samples were minced into pieces of approximately $0.5 \mathrm{~mm}^{2}$ and digested for $1.5 \mathrm{~h}$ at $37^{\circ} \mathrm{C}$ using $0.2 \%$ type I collagenase (Sigma-Aldrich, St. Louis, MO). The minced/digested LF tissue samples were washed with Dulbecco's Modified Eagle's medium (DMEM, Gibco, New York, NY) and were centrifuged 3 times (1000r/min, $5 \mathrm{~min}$ ). Finally, the LF tissue samples were placed in 6-well plates (Corning-Costar, New York, NY) in DMEM supplemented with 10\% fetal bovine serum (Gibco), $100 \mathrm{U} / \mathrm{ml}$ penicillin and $100 \mathrm{mg} / \mathrm{ml}$ streptomycin (Gibco) and incubated in a $5 \%$ $\mathrm{CO}_{2}$ humidified incubator at $37^{\circ} \mathrm{C}$. The medium was changed every 3 days. After approximately 10 days, LF cells started to migrate out of the LF tissue samples and formed a monolayer. At $90 \%$ confluence, LF cells were passaged 1:3.

The morphology of LF cells was observed under a phase contrast microscope (Axio Scope A1, Zeiss, Oberkochen, Germany). The expression of collagen I and vimentin was detected via immunostaining (see above) and was observed under a confocal microscope (FV1200, Olympus, Tokyo, Japan) to identify the cell type. LF cells of the third passage were treated with different concentrations of recombinant human IGF-1 


\section{Cellular Physiology Cell Physiol Biochem 2018;48:293-303 \begin{tabular}{l|l} 
and Biochemistry Published & $\begin{array}{l}\text { DOI: 1159/000491729 } \\
\text { a } 2018 \text { The Author(s). Published by S. Karger AG, Basel } \\
\text { www.karger.com/cpb }\end{array}$
\end{tabular}}

Yan et al.: IGF-1 Promotes Hypertrophy of the Ligamentum Flavum

$(0,1,10,100 \mathrm{ng} / \mathrm{ml}$, Sino Biological, Beijing, China) with or without $100 \mathrm{ng} / \mathrm{ml}$ NVP-AEW541 (MedChen Express, Monmouth Junction, NJ) or $10 \mathrm{ng} / \mathrm{ml}$ rapamycin (Alexis Biochemicals, Lausen, Switzerland) for $24 \mathrm{~h}$.

\section{Cell viability assays}

The viability of LF cells was evaluated using the 3-(4, 5-dimethylthiazol-2-y1)-2, 5-diphenyltetrazolium bromide colorimetric assay (MTT, Solarbio, Beijing, China). LF cells were seeded in 96-well plates at $1 \times 10^{4}$ cells per well and were incubated at $37^{\circ} \mathrm{C}$ under $5 \% \mathrm{CO}_{2}$ for $24 \mathrm{~h}$. LF cells were washed with PBS 2 times and incubated in $90 \mu \mathrm{l}$ of DMEM with $10 \mu \mathrm{l}$ of MTT solution in each well for $4 \mathrm{~h}$. After medium removal, $110 \mu \mathrm{l}$ of dimethyl sulfoxide (DMSO) was added to each well to dissolve formazan, and the absorbance of the solution was measured at $490 \mathrm{~nm}$ using a multi-mode reader (HTX, Gene, Shanghai, China).

\section{Western blotting assays}

LF tissues and LF cells were lysed in Laemmli buffer (62.5 mM Tris-HCl, pH 6.8, 2\% sodium dodecyl sulfate, $10 \%$ glycerol, $50 \mathrm{mM}$ dithiothreitol, and $0.01 \%$ bromophenol blue) for 10 minutes at $100{ }^{\circ} \mathrm{C}$. The tissue and cell lysates were separated via $8 \%$ or $15 \%$ sodium dodecyl sulfate polyacrylamide gel electrophoresis and transferred to nitrocellulose membranes (Millipore, Billerica, MA). The membranes were blocked with $5 \%$ non-fat milk diluted in Tris-buffered saline for $2 \mathrm{~h}$ at room temperature. Next, the membranes were incubated at $4^{\circ} \mathrm{C}$ overnight with primary antibodies as follows: collagen I (1:1000, Abcam), collagen III (1:1000, Abcam), IGF-1(1:1000, Abcam), pIGF-1R (1:1000, Abcam), IGF-1R (1:1000, Abcam), pAKT (1:1000, Abcam), AKT (1:1000, Abcam), pS6 (1:100, ABclonal, Boston, MA), S6 (1:1000, ABclonal), and $\beta$-actin (1:1000, ABclonal). Next, the blots were washed and incubated with anti-rabbit or anti-mouse immunoglobulin G peroxidase conjugate (1:3000, RayBiotech, Beijing, China) for $1.5 \mathrm{~h}$ at room temperature. The signals were detected using an enhanced chemiluminescence kit (Beyotime, Shanghai, China).

\section{Statistical Analysis}

The independent-sample t-test was used to compare the Non-LSS group with the LSS group in terms of age, LF thickness and grade of LF fibrosis (IBM SPSS Statistics 22.0, SPSS, Chicago, IL). One-way ANOVA was used to analyze the MTT assay results, and unpaired Student's t-test was used to determine significant differences in the immunohistochemical staining data (GraphPad Prism 5.01, La Jolla, CA). The results are presented as the mean \pm SD for all parameters measured. $\mathrm{P}<0.05$ was considered statistically significant.

\section{Results}

Ligamentum flavum thickness and fibrosis of the Non-LSS group and LSS group

The ligamentum flavum thickness of each patient was measured via MRI, and the results showed that the mean ligamentum flavum thickness in the Non-LSS group $(2.84 \pm 0.47 \mathrm{~mm})$ was significantly lower than that in the LSS group $(5.76 \pm 0.57 \mathrm{~mm}$ ) (Table 1). Meanwhile, the degree of ligamentum flavum fibrosis was higher in the LSS group compared to that in the Non-LSS group (Table 1). Histological examinations of H\&E (Fig. 2 A, B) and Masson's trichrome (Fig. 2 C, D) stained sections showed a significant loss of elastic fibers and an increase in collagen fibers in the LSS group and a lack of obvious alterations in the Non-LSS group.

Ultrastructure of LF tissues in the
Non-LSS group and LSS group
The transmission electron microscopy (TEM) results showed that the LF tissues in the Non-LSS group primarily contained elastic fibers, with very few collagen fibers among them (Fig. $3 \mathrm{~A}$ ), and that the number of elastic fibers was reduced significantly and number of collagen fibers was markedly increased in
Table 1. General data of patients in two groups. Independent-sample t-test; data are presented as the mean $\pm \mathrm{SD}$; $\mathrm{P}<0.05$ is considered to be significant. $\mathrm{LF}$, ligamentum flavum; LSS, lumbar spinal stenosis

\begin{tabular}{lccr}
\hline & Non-LSS Group $(\mathrm{n}=20)$ & LSS Group $(\mathrm{n}=20)$ & P value \\
\hline Age (years) & $56.05 \pm 9.24$ & $59.60 \pm 7.80$ & 0.197 \\
LF thickness (mm) & $2.84 \pm 0.47$ & $5.76 \pm 0.57$ & $<0.001$ \\
Grade of LF fibrosis & $0.2 \pm 0.41$ & $3.1 \pm 0.79$ & $<0.001$ \\
Gender & 8 males,12 females & 10 males,10 females & \\
Lumbar level & L4/5 & L4/5 & \\
\hline
\end{tabular}




\section{Cellular Physiology Cell Physiol Biochem 2018;48:293-303 \begin{tabular}{l|l} 
DOI: 10.1159/000491729 & $\begin{array}{l}\text { O } 2018 \text { The Author(s). Published by S. Karger AG, Basel } \\
\text { www.karger.com/cpb }\end{array}$
\end{tabular}}

Fig. 2. Morphology and fibrosis of ligamentum flavum tissues in the Non-LSS group and LSS group. Representative images of H\&E staining of the Non-LSS group (A) and LSS group (B), with elastic fibers stained crimson and collagen fibers stained pink; representative images of Masson's trichrome staining of the NonLSS group (C) and LSS group (D), with elastic fibers stained red and collagen fibers stained blue; representative images of collagen I immunohistochemical staining (brown stain) of the Non-LSS group (E) and LSS group (F); representative images of collagen III immunohistochemical staining (brown stain) of the Non-LSS group (G) and LSS group (H). The scale bar indicates $50 \mu \mathrm{m}$.

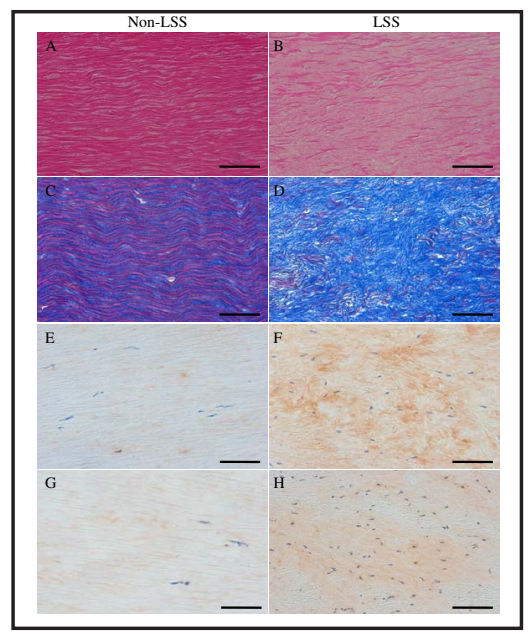

Fig. 3. Transmission electron microscopy examination of ligamentum flavum tissues of the Non-LSS group and LSS group. In the Non-LSS group, oval elastic fibers form the majority, with few collagen fibers among them (A); inactive ligamentum flavum cells contain a small amount of cytoplasm and organelles (B). In the LSS group, oval elastic fibers are reduced and collagen fibers are increased in number (C), and active ligamentum flavum cells have an abundant cytoplasm and more organelles (D). EF: elastic fibers; CF: collagen fibers; LFC: ligamentum flavum cell; blue arrows point to the mitochondria, and red arrows point to the rough endoplasmic

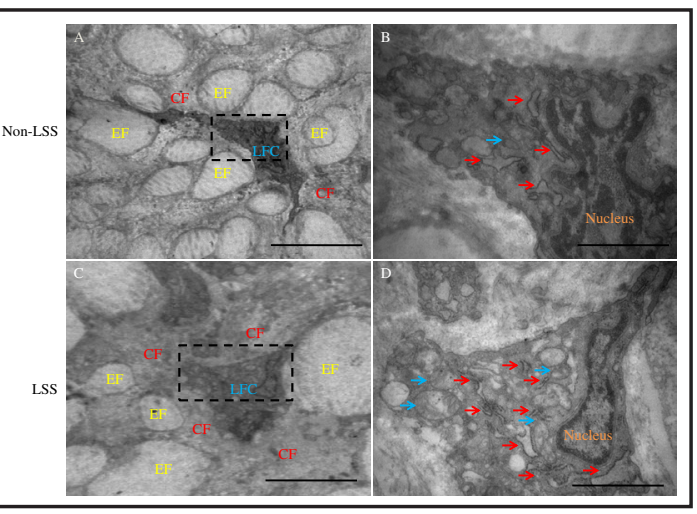
reticulum. (A) and (C): 8000×; (B) and (D): 40000×; the scale bar indicates $5 \mu \mathrm{m}$ in (A) and (C) and $1 \mu \mathrm{m}$ in (B) and (D).

the LSS group (Fig. 3 C). At $40000 \times$ magnification, inactive ligamentum flavum cells were found to have a small number of cytoplasm with few mitochondria and a rough endoplasmic reticulum in the Non-LSS group (Fig. 3 B), while active ligamentum flavum cells were found to have an abundant cytoplasm with a large number of mitochondria and a rough endoplasmic reticulum in the LSS group (Fig. 3 D).

High levels of IGF-1, pIGF-1R, pAKT, pS6, collagen I and collagen III in the LSS group

To examine the potential involvement of IGF-1 signaling in LSS, immunohistochemical staining and Western blot analyses were performed to analyze the expression of IGF-1, pIGF1R, pAKT, pS6, collagen I and collagen III in LF tissues of the two groups. Compared to the Non-LSS group, the number of cells positive for IGF-1 (Fig. 4 A1, A2, A3, A4, A5), pIGF-1R (Fig. 4 B1, B2, B3, B4, B5), pAKT (Fig. 4 C1, C2, C3, C4, C5) and pS6 (Fig. 4 D1, D2, D3, D4, D5) were significantly elevated in the LSS group. Meanwhile, the expression of collagen I (Fig. 2 E, F) and collagen III (Fig. 2 G, H) was obviously higher in the LSS group than that in the Non-LSS group. In addition, as shown by Western blot analyses, remarkable increases were observed in the protein expression levels of IGF-1, pIGF-1R, pAKT, pS6, collagen I and collagen III in the LSS group compared to those in the Non-LSS group (Fig. 5).

IGF-1 stimulated mTORC1 activation, collagen I and collagen III expression in LF cells

In vitro studies with primary LF cells were conducted to investigate the involvement of IGF1 and the mTORC1 signaling pathway in the HLF process. As shown by immunofluorescence staining (Fig. 6 A, B), most cells were labeled with collagen I or vimentin (markers of LF 
Fig. 4. Immunohistochemical staining of IGF-1, pIGF-1, pAKT and pS6 in ligamentum flavum tissues. Images of immunostaining of IGF-1 in the Non-LSS group (A1, A2) and LSS group (A3, A4); pIGF-1R in the NonLSS group (B1, B2) and LSS group (B3, B4); pAKT in the Non-LSS group (C1, C2) and LSS group (C3, C4); pS6 in the Non-LSS group (D1, D2) and LSS group (D3, D4). Quantitative analyses of cells positive for IGF-1 (A5), pIGF-1R (B5), pAKT (C5) and pS6 (D5) (arrows indicate positive cells; scale bar $=50 \mu \mathrm{m}$; ${ }^{* * *}$ indicates $\left.\mathrm{P}<0.001\right)$.

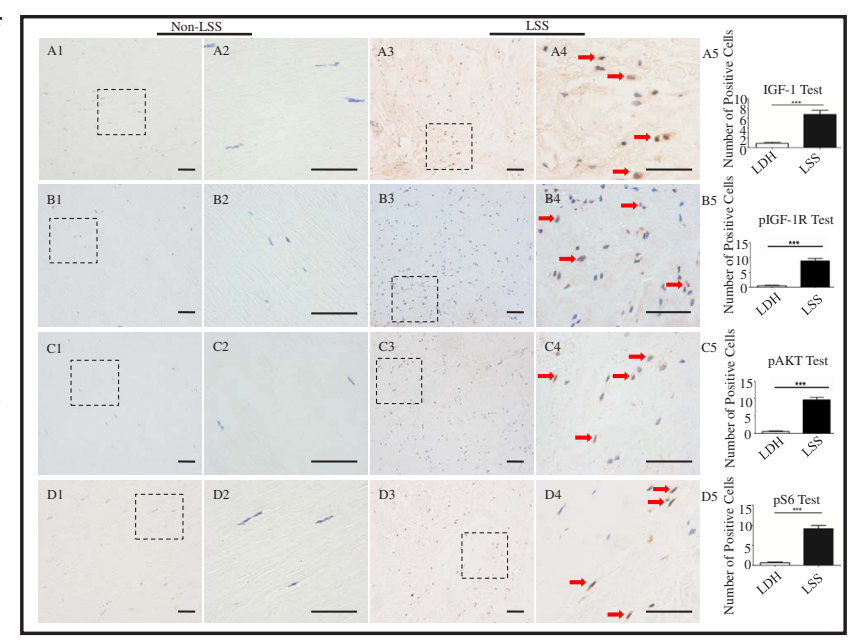

Fig. 5. Protein expression levels of IGF-1, pIGF-1R, pAKT, pS6, collagen I and collagen III in ligamentum flavum tissues. Levels of IGF-1, pIGF-1R, pAKT, pS6, collagen I and collagen III are obviously higher in the LSS group than those in the Non-LSS group.

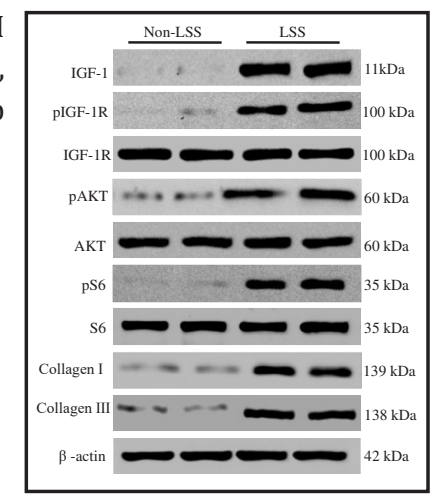

Fig. 6. Identification and viability test of ligamentum flavum cells. Immunofluorescence staining of collagen I (A) and vimentin (B); quantitative analyses of the MTT assay on ligamentum flavum cells cultured with different concentrations of IGF-1, showing no obvious alterations of cell viability $(C, P=0.58)$. Immunofluorescence was

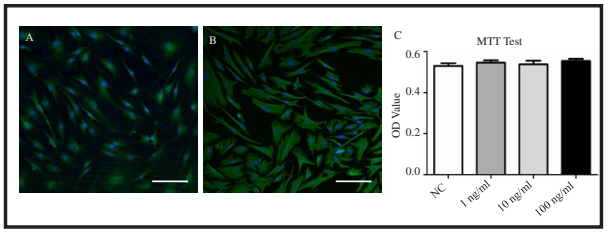
visualized using FITC (Green), and nuclei were visualized using DAPI (Blue); scale bar $=50 \mu \mathrm{m}$.

cells), suggesting a high purity of LF cells was obtained. In addition, no apparent alteration of cell viability was found in LF cells after being exposed to different concentrations of IGF-1 (Fig. 6 C, P=0.58), suggesting that IGF-1 exposure exhibited no toxicity in LF cells.

As shown in Fig. 7 and Fig. 8, the protein expression levels of pIGF-1R, pAKT, pS6, collagen I and collagen III in LF cells increased significantly in a dose-dependent manner after exposure to exogenous IGF-1.

Both NVP-AEW541 and rapamycin can block IGF-1-induced collagen I and collagen III overexpression in LF cells

To confirm the role of mTORC1 signaling in IGF-1-induced collagen I and collagen III expression in LF cells, the IGF-1R specific inhibitor NVP-AEW541 and mTORC1 specific inhibitor rapamycin were included in the exogenous IGF-1 exposure experiments. After treatment with $100 \mathrm{ng} / \mathrm{ml}$ NVP-AEW541, IGF-1-induced pIGF-1R, pAKT, pS6, collagen I and collagen III overexpression was significantly attenuated in LF cells (Fig. 7). Similarly, after treatment with $10 \mathrm{ng} / \mathrm{ml}$ rapamycin, IGF-1-induced pS6, collagen I and collagen III overexpression was obviously decreased (Fig. 8) in LF cells.

\section{KARGER}




\section{Cellular Physiology and Biochemistry \\ Cell Physiol Biochem 2018;48:293-303 \\ \begin{tabular}{l|l}
\hline DOI: $10.1159 / 000491729$ & (C) 2018 The Author(s). Published by S. Karger AG, Basel
\end{tabular} \\ Published online: July 16, 2018 www.karger.com/cpb}

Fig. 7. Protein expression levels of pIGF-1R, pAKT, pS6, collagen I and collagen III in ligamentum flavum cells after exposure to exogenous IGF-1 with or without the IGF-1R inhibitor NVP-AEW541. Expression of pIGF-1R, pAKT, pS6, collagen I and collagen III in ligamentum flavum cells increased significantly and in a dose-dependent manner after exposure to various concentrations of exogenous IGF1 without NVP-AEW541; IGF-1-induced increases in the expression of pIGF-1R, pAKT, pS6, collagen I and collagen III were attenuated significantly with NVP-

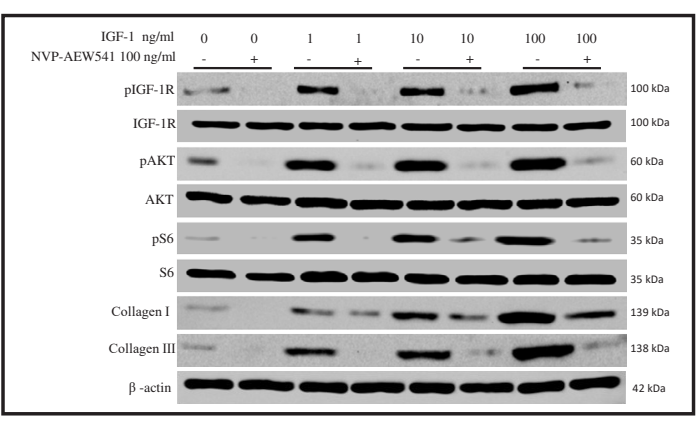
AEW541.

Fig. 8. Protein expression levels of pS6, collagen I and collagen III in ligamentum flavum cells after exposure to various concentrations of exogenous IGF-1 with or without rapamycin. Levels of pS6, collagen I and collagen III increased significantly and in a dose-dependent manner after IGF-1 exposure without rapamycin; IGF-1-induced overexpression of pS6, collagen I and collagen III was attenuated

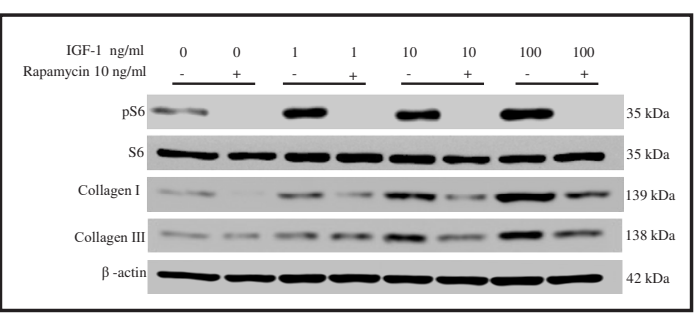
significantly with rapamycin.

\section{Discussion}

HLF is a major pathogenic factor for LSS. LSS can lead to nerve root compression, low back pain and intermittent claudication and can even develop into paralysis [4-6]. Patients with LSS usually suffer from these symptoms for many years, which cause significant morbidity. Previously, numerous studies have investigated the pathological process of HLF, which ultimately results in LSS; however, the molecular basis of HLF remains largely unknown. The normal ligamentum flavum, anatomically, is composed of $80 \%$ elastic fibers and $20 \%$ collagen fibers [30]. In HLF, elastic fibers gradually crack, become disordered and reduce in number. On the other hand, collagen fibers are constantly produced and accumulate and contribute to HLF [5]. According to previous reports [13-15], the increased collagen fibers are mainly collagen I and collagen III. As collagen I and collagen III production is believed to strongly correlate with HLF [14, 31, 32], in our study, we measured collagen I and collagen III expression in LF tissues of the Non-LSS group and LSS group. In the current study, the expression of collagen I and collagen III in LF tissues was found to be increased significantly and was associated with the increases in LF tissue thickness and degree of fibrosis in the LSS group compared with the Non-LSS group.

To characterize HLF further, ultrastructural comparisons of LF tissues between the two groups were performed by transmission electron microscopy. Our data suggest that there was increased deposition of collagen fibers in the LSS group than in the Non-LSS group. Furthermore, compared to the Non-LSS group, ligamentum flavum cells in the LSS group were found to have an abundant cytoplasm and more mitochondrion and a rough endoplasmic reticulum. Since protein is synthesized in ribosomes on the rough endoplasmic reticulum and the mitochondria provide energy for this process, from an energy metabolism perspective, ligamentum flavum cells have a higher energy metabolism level in the LSS group than in the Non-LSS group, which can explain the increased production of abundant collagen fibers in the LSS group compared to that in the Non-LSS group.

To explore the potential pathophysiological mechanisms of HLF, many investigators have focused on investigating the relationship between inflammatory factors and HLF. Some 
studies suggested that chronic inflammation induced by mechanical stress was a vital impact factor for HLF $[11,12]$. It is believed that when suffering from increased mechanical stress, the ligamentum flavum is constantly damaged, causing a local release of inflammationrelated factors, such as TNF- $\alpha$, TGF- $\beta 1$, IL- 1 and IL- 6 , which can stimulate LF fibrosis and contribute to the pathological process of HLF [12,33-36]. Although inflammatory factors are believed to play a crucial role in HLF [5, 11,37-40], the molecular basis for LF tissue fibrosis remains unclear.

As IGF-1 has previously been found to be a significant growth factor that plays a critical role in the fibrosis process in many tissues [18-20], the current study focused on investigating the potential role of IGF- 1 in HLF/LF tissue fibrosis and the molecular mechanism potentially involved. Under mechanical loading, IGF-1 has been previously found to be released by various types of cells, which then induces the production of collagen fibers and, ultimately, results in fibrosis and hypertrophy of different tissues [23-27]. Furthermore, IGF-1 is known to specifically activate mTORC1 signaling and initiate its physiological functions in mammals [18-20,41-44], and to date, no studies have revealed the potential functions of IGF-1 and mTORC1 signaling in HLF; therefore, the current study hypothesized that IGF-1, through mTORC1 signaling, may contribute to the pathological process of HLF by activating collagen fiber production. Thus, to test our hypothesis, we first performed immunohistochemical analyses of LF tissues, which were significantly increased in LF cells that positively express IGF-1, pIGF-1R, pAKT (the specific upstream marker of mTORC1 signaling) and pS6 (the specific downstream marker of mTORC1 signaling) in the LSS group; these markers were expressed in only a few cells in the Non-LSS group. Furthermore, the Western blot analysis results of LF tissues showed a remarkable increase in the protein expression levels of IGF-1, pIGF-1R, pAKT, pS6, collagen I and collagen III in the LSS group compared to those in the NonLSS group. These data suggest that a high local expression of IGF-1 in the ligamentum flavum of the LSS group upregulates the phosphorylation of its receptor (IGF-1R) and activates the downstream mTORC1 signaling pathway to enhance collagen I and collagen III production.

To further verify our hypothesis and investigate the potential molecular mechanism involved in the IGF-1 action, we conducted in vitro studies on isolated primary LF cells. In 2009, Zhong et al [45]. established a primary culture of human LF cells and distinguished them based on the expression of collagen I and vimentin. Thus, following primary culture, collagen I and vimentin expression was used to identify the purity of the LF cells used in the current study. Our immunofluorescence results showed that the LF cells we isolated exhibited good purity.

Furthermore, while previous studies have reported that both IGF-1 and mTORC1 signaling pathways can stimulate the production of collagen fibers [23-27], it is unknown whether there is a clear interconnection between these two pathways in HLF. Our in vitro studies in LF cells showed that exogenous IGF-1 treatment induced a dose-dependent increase of collagen I and collagen III by activating the mTORC1 signaling pathway. Moreover, we found that both NVP-AEW541 (IGF-1R specific inhibitor) and rapamycin (mTORC1 specific inhibitor) blocked IGF-1-induced collagen I and collagen III overexpression in LF cells. These results suggest that the mTORC1 signaling pathway may play a vital mediatory role in IGF-1-induced collagen I and collagen III overexpression in the pathological process of HLF (Fig. 9).

\section{Conclusion}

In summary, in this study, we found a high IGF-1 expression level and relevant signaling pathway in HLF. Our study suggested that IGF-1 might contribute to the HLF process by promoting the production of collagen I and collagen III. Moreover, for the HLF process, the mediatory role of the mTORC1 signaling pathway for IGF-1-induced collagen I and collagen III overexpression was also verified. Thus, the current study concluded that locally produced IGF-1 contributed to HLF via the mTORC1 signaling pathway. These findings provide a 


\section{Cellular Physiology Cell Physiol Biochem 2018;48:293-303 \begin{tabular}{l|l} 
and Biochemistry Published onlIne: July 16, 2018 & $\begin{array}{l}\text { (c) } 2018 \text { The Author(s). Published by S. Karger AG, Basel } \\
\text { www.karger.com/cpb }\end{array}$ \\
\hline
\end{tabular}}

Yan et al.: IGF-1 Promotes Hypertrophy of the Ligamentum Flavum

Fig. 9. Potential signaling pathway involving mTORC1 activation for IGF-1-induced accumulation of collagen fibers in ligamentum flavum cells.Locally produced IGF-1 binds to the IGF-1 receptor on the membrane of ligamentum flavum cells and further activates the downstream mTORC1 signaling pathway, which promotes the production of collagen I and collagen III and, eventually, contributes to HLF.

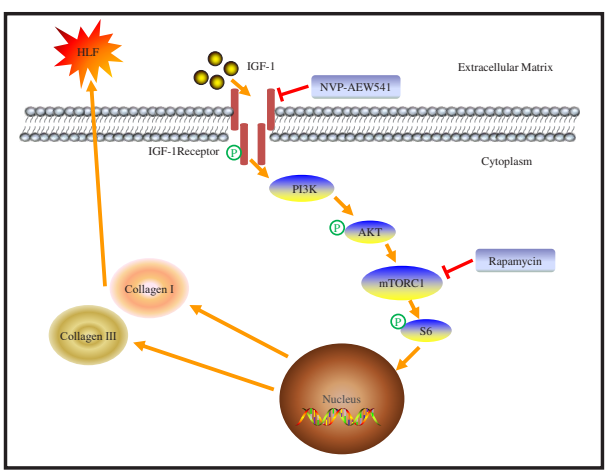

novel aspect for understanding the pathogenesis of HLF. Due to the absence of HLF animal models, in the current study, we did not evaluate whether IGF-1 inhibition attenuated the HLF process in vivo. Therefore, one important component of our further work is to establish an HLF animal model and to verify IGF-1 action in the HLF process in vivo.

\section{Acknowledgements}

This work was supported by the National Natural Science Foundation of China (No.31370985; 81672228; 81702199) and Natural Science Foundation of Guangdong Province (No.2015A030310416).

\section{Disclosure Statement}

All authors state that they have no conflict of interests.

\section{References}

-1 Altun I, Yüksel KZ: Histopathological analysis of ligamentum flavum in lumbar spinal stenosis and disc herniation. Asian Spine J 2017;11:71-74.

-2 Backstrom KM, Whitman JM, Flynn TW: Lumbar spinal stenosis-diagnosis and management of the aging spine. Man Ther 2011;16:308-317.

-3 Yabe Y, Hagiwara Y, Tsuchiya M, Honda M, Hatori K, Sonofuchi K, Kanazawa K, Koide M, Sekiguchi T, Itaya $\mathrm{N}$, Itoi E: Decreased elastic fibers and increased proteoglycans in the ligamentum flavum of patients with lumbar spinal canal stenosis. J Orthop Res 2016;34:1241-1247.

-4 Munns JJ, Lee JY, Espinoza Orias AA, Takatori R, Andersson GB, An HS, Inoue N: Ligamentum flavum hypertrophy in asymptomatic and chronic low back pain subjects. PLoS One 2015;10:e128321.

5 Sairyo K, Biyani A, Goel V, Leaman D, Booth R, Jr., Thomas J, Gehling D, Vishnubhotla L, Long R, Ebraheim N: Pathomechanism of ligamentum flavum hypertrophy: A multidisciplinary investigation based on clinical, biomechanical, histologic, and biologic assessments. Spine 2005;30:2649-2656.

6 Szpalski M, Gunzburg R: Lumbar spinal stenosis in the elderly: An overview. Eur Spine J 2003;12:S170-175.

7 Cheung PWH, Tam V, Leung VYL, Samartzis D, Cheung KM, Luk KD, Cheung JPY: The paradoxical relationship between ligamentum flavum hypertrophy and developmental lumbar spinal stenosis. Scoliosis Spinal Disord 2016;11:26.

-8 Sakai Y, Ito S, Hida T, Ito K, Harada A, Watanabe K: Clinical outcome of lumbar spinal stenosis based on new classification according to hypertrophied ligamentum flavum. J Orthop Sci 2017;22:27-33.

-9 Kim YU, Park JY, Kim DH, Karm MH, Lee JY, Yoo JI, Chon SW, Suh JH: The role of the ligamentum flavum area as a morphological parameter of lumbar central spinal stenosis. Pain physician 2017;20:E419-424.

10 Costandi S, Chopko B, Mekhail M, Dews T, Mekhail N: Lumbar spinal stenosis: Therapeutic options review. Pain Pract 2015;15:68-81. 


\section{Cellular Physiology Cell Physiol Biochem 2018;48:293-303 \begin{tabular}{l|l} 
DOI: 10.1159/000491729 & $\begin{array}{l}\text { O } 2018 \text { The Author(s). Published by S. Karger AG, Basel } \\
\text { www.karger.com/cpb }\end{array}$
\end{tabular}}

Yan et al.: IGF-1 Promotes Hypertrophy of the Ligamentum Flavum

-11 Sairyo K, Biyani A, Goel VK, Leaman DW, Booth R, Jr., Thomas J, Ebraheim NA, Cowgill IA, Mohan SE: Lumbar ligamentum flavum hypertrophy is due to accumulation of inflammation-related scar tissue. Spine 2007;32:E340-347.

12 Park JO, Lee BH, Kang YM, Kim TH, Yoon JY, Kim H, Kwon UH, Lee KI, Lee HM, Moon SH: Inflammatory cytokines induce fibrosis and ossification of human ligamentum flavum cells. J Spinal Disord Tech 2013;26:E6-12.

13 Nakatani T, Marui T, Hitora T, Doita M, Nishida K, Kurosaka M: Mechanical stretching force promotes collagen synthesis by cultured cells from human ligamentum flavum via transforming growth factor-beta1 J Orthop Res 2002;20:1380-1386.

14 Zhong Z-M, Zha D-S, Xiao W-D, Wu S-H, Wu Q, Zhang Y, Liu F-Q, Chen J-T: Hypertrophy of ligamentum flavum in lumbar spine stenosis associated with the increased expression of connective tissue growth factor. J Orthop Res 2011;29:1592-1597.

15 Zhang Y, Chen J, Zhong ZM, Yang D, Zhu Q: Is platelet-derived growth factor-bb expression proportional to fibrosis in the hypertrophied lumber ligamentum flavum? Spine 2010;35:E1479-1486.

16 Hur JW, Kim B-J, Park J-H, Kim J-H, Park Y-K, Kwon T-H, Moon HJ: The mechanism of ligamentum flavum hypertrophy. Neurosurgery 2015;77:274-282.

17 Okuda T, Baba I, Fujimoto Y, Tanaka N, Sumida T, Manabe H, Hayashi Y, Ochi M: The pathology of ligamentum flavum in degenerative lumbar disease. Spine 2004;29:1689-1697.

18 Cui H, Wang L, Yu Y: Production and characterization of alkaline protease from a high yielding and moderately halophilic strain of SD11 marine bacteria. J Chem 2015;2015:1-8.

19 Xian L, Wu X, Pang L, Lou M, Rosen CJ, Qiu T, Crane J, Frassica F, Zhang L, Rodriguez JP, Jia X, Yakar S, Xuan S, Efstratiadis A, Wan M, Cao X: Matrix IGF-1 maintains bone mass by activation of mtor in mesenchymal stem cells. Nat Med 2012;18:1095-1101.

20 Carrasco L, Cea P, Rocco P, Peña-Oyarzún D, Rivera-Mejias P, Sotomayor-Flores C, Quiroga C, Criollo A, Ibarra C, Chiong M, Lavandero S: Role of heterotrimeric g protein and calcium in cardiomyocyte hypertrophy induced by IGF-1. J Cell Biochem 2014;115:712-720.

-21 Reijnders CMA, Bravenboer N, Tromp AM, Blankenstein MA, Lips P: Effect of mechanical loading on insulinlike growth factor-1 gene expression in rat tibia. J Endocrinol 2007;192:131-140.

-22 Juffer P, Jaspers RT, Lips P, Bakker AD, Klein-Nulend J: Expression of muscle anabolic and metabolic factors in mechanically loaded MLO-Y4 osteocytes. Am J Physiol Endocrinol Metab 2012;302:E389-395.

23 Adams GR, McCue SA: Localized infusion of IGF-1 results in skeletal muscle hypertrophy in rats. J Appl Physiol 1998;84:1716-1722.

24 Blackstock CD, Higashi Y, Sukhanov S, Shai S-Y, Stefanovic B, Tabony AM, Yoshida T, Delafontaine P: Insulin-like growth factor-1 increases synthesis of collagen type I via induction of the mRNA-binding protein LARP6 expression and binding to the $5^{\prime}$ stem-loop of COL1a1 and COL1a2 mRNA. J Biol Chem 2014;289:7264-7274.

25 Doessing S, Holm L, Heinemeier KM, Feldt-Rasmussen U, Schjerling P, Qvortrup K, Larsen JO, Nielsen RH, Flyvbjerg A, Kjaer M: GH and IGF1 levels are positively associated with musculotendinous collagen expression: Experiments in acromegalic and gh deficiency patients. Eur J Endocrinol 2010;163:853-862.

-26 Honsho S, Nishikawa S, Amano K, Zen K, Adachi Y, Kishita E, Matsui A, Katsume A, Yamaguchi S, Nishikawa K, Isoda K, Riches DWH, Matoba S, Okigaki M, Matsubara H: Pressure-mediated hypertrophy and mechanical stretch induces IL-1 release and subsequent IGF-1 generation to maintain compensative hypertrophy by affecting Akt and JNK pathways. Circ Res 2009;105:1149-1158.

-27 Li P, Liang ML, Zhu Y, Gong YY, Wang Y, Heng D, Lin L: Resveratrol inhibits collagen I synthesis by suppressing IGF-1R activation in intestinal fibroblasts. World J Gastroenterol 2014;20:4648-4661.

28 Kolte VS, Khambatta S, Ambiye MV: Thickness of the ligamentum flavum: Correlation with age and its asymmetry-an magnetic resonance imaging study. Asian Spine J 2015;9:245-253.

29 Sakamaki T, Sairyo K, Sakai T, Tamura T, Okada Y, Mikami H: Measurements of ligamentum flavum thickening at lumbar spine using mri. Arch Orthop Trauma Surg 2009;129:1415-1419.

-30 Safak AA, Is M, Sevinc O, Barut C, Eryoruk N, Erdogmus B, Dosoglu M: The thickness of the ligamentum flavum in relation to age and gender. Clin Anat 2010;23:79-83.

-31 Kosaka H, Sairyo K, Biyani A, Leaman D, Yeasting R, Higashino K, Sakai T, Katoh S, Sano T, Goel VK, Yasui $\mathrm{N}$ : Pathomechanism of loss of elasticity and hypertrophy of lumbar ligamentum flavum in elderly patients with lumbar spinal canal stenosis. Spine 2007;32:2805-2811. 


\section{Cellular Physiology Cell Physiol Biochem 2018;48:293-303 \begin{tabular}{l|l} 
DOI: 10.1159/000491729 & $\begin{array}{l}\text { O 2018 The Author(s). Published by S. Karger AG, Basel } \\
\text { www.karger.com/cpb }\end{array}$
\end{tabular}

-32 Yabe Y, Hagiwara Y, Ando A, Tsuchiya M, Minowa T, Takemura T, Honda M, Hatori K, Sonofuchi K, Kanazawa $\mathrm{K}$, Koide M, Sekiguchi T, Itoi E: Chondrogenic and fibrotic process in the ligamentum flavum of patients with lumbar spinal canal stenosis. Spine 2015;40:429-435.

33 Kim B-J, Hur JW, Park JS, Kim JH, Kwon T-H, Park Y-K, Moon HJ: Expression of matrix metalloproteinase-2 and -9 in human ligamentum flavum cells treated with tumor necrosis factor- $\alpha$ and interleukin-1 $\beta$. J Neurosurg Spine 2016;24:428-435.

-34 Lakemeier S, Schofer MD, Foltz L, Schmid R, Efe T, Rohlfs J, Fuchs-Winkelmann S, El-Zayat BF, Paletta JR, Foelsch C: Expression of hypoxia-inducible factor-1alpha, vascular endothelial growth factor, and matrix metalloproteinases 1, 3, and 9 in hypertrophied ligamentum flavum. J Spinal Disord Tech 2013;26:400-406.

-35 Löhr M, Hampl JA, Lee JY, Ernestus R-I, Deckert M, Stenzel W: Hypertrophy of the lumbar ligamentum flavum is associated with inflammation-related TGF- $\beta$ expression. Acta Neurochir 2010;153:134-141.

36 Cao Y-L, Duan Y, Zhu L-X, Zhan Y-N, Min S-X, Jin A-M: TGF- $\beta 1$, in association with the increased expression of connective tissue growth factor, induce the hypertrophy of the ligamentum flavum through the p38 MAPK pathway. Int J Mol Med 2016;38:391-398.

37 Morishita R, Nakamura T, Okada T, Endo M, Kadomatsu T, Taniwaki T, Sei A, Odagiri H, Masuda T, Fujimoto T, Nakamura T, Oike Y, Mizuta H: Angiopoietin-like protein 2 induced by mechanical stress accelerates degeneration and hypertrophy of the ligamentum flavum in lumbar spinal canal stenosis. PLoS ONE 2014;9:e85542.

-38 Park J-B, Kong C-G, Suhl K-H, Chang E-D, Riew KD: The increased expression of matrix metalloproteinases associated with elastin degradation and fibrosis of the ligamentum flavum in patients with lumbar spinal stenosis. Clin Orthop Surg 2009;1:81-89.

39 Park JB, Chang H, Lee JK: Quantitative analysis of transforming growth factor-beta 1 in ligamentum flavum of lumbar spinal stenosis and disc herniation. Spine 2001;26:E492-495.

-40 Hur JW, Bae T, Ye S, Kim JH, Lee S, Kim K, Lee SH, Kim JS, Lee JB, Cho TH, Park JY, Hur JK: Myofibroblast in the ligamentum flavum hypertrophic activity. Eur Spine J 2017;26:2021-2030.

-41 McMullen JR, Izumo S: Role of the insulin-like growth factor 1 (IGF1)/phosphoinositide-3-kinase (PI3K) pathway mediating physiological cardiac hypertrophy. Novartis Found Symp 2006;274:90-111.

-42 Mata R, Palladino C, Nicolosi ML, Lo Presti AR, Malaguarnera R, Ragusa M, Sciortino D, Morrione A, Maggiolini M, Vella V, Belfiore A: IGF-1 induces upregulation of DDR1 collagen receptor in breast cancer cells by suppressing MIR-199a-5p through the PI3K/AKT pathway. Oncotarget 2016;7:7683-7700.

43 Bakker AD, Gakes T, Hogervorst JMA, de Wit GMJ, Klein-Nulend J, Jaspers RT: Mechanical stimulation and IGF-1 enhance mRNA translation rate in osteoblasts via activation of the AKT-mTOR pathway. J Cell Physiol 2016;231:1283-1290.

44 Sheng MHC, Lau KHW, Baylink DJ: Role of osteocyte-derived insulin-like growth factor 1 in developmental growth, modeling, remodeling, and regeneration of the bone. J Bone Metab 2014;21:41-54.

45 Zhong Z-M, Chen J-T: Phenotypic characterization of ligamentum flavum cells from patients with ossification of ligamentum flavum. Yonsei Med J 2009;50:375-379. 